\title{
3D SPATIAL ATTENTION EFFECTS ARE INDEPENDENT OF PROJECTED 2D SIZE AND LOCATION FOR OLDER AND YOUNGER DRIVERS
}

\author{
Russell S. Pierce \& George J. Andersen \\ Department of Psychology, University of California, Riverside \\ Riverside, California, USA \\ Email: Russell.Pierce@dot.gov
}

\begin{abstract}
Summary: Previous research has found the reaction time (RT) to light change targets when performing a car following task, is partially dependent on the distance in depth of the target from the driver. Researchers have concluded from this evidence that the spatial extent of attention is three dimensional (3D) during dual-task driving. However, in prior experiments the effect of two-dimensional (2D) projected size and position was not experimentally controlled. If spatial attention is 3D, then there should be an effect of target distance when 2D projected size and position are constant. The purpose of the current work was to assess this hypothesis. We manipulated the size and position of light-change targets at different depths to hold the projected size and position of targets constant between blocks. Although projected size and position were identical in this experiment, the results demonstrated that for younger and older drivers, targets further from the driver were responded to more slowly than targets closer to the driver. These results demonstrate that 3D attention effects are not dependent on projected size or position, and that the mechanism of $3 \mathrm{D}$ attention is present in younger and older drivers. These results, considered with the findings of other studies, suggest that tests to assess crash risk, such as the UFOV, are limited in scope because such tests fail to incorporate variation in attention as a function of distance.
\end{abstract}

\section{OBJECTIVES}

Per mile driven, older drivers have a greater than average crash risk (Tefft, 2008). Researchers have claimed that this is partially due to reductions in the useful field of view (UFOV), (i.e. spatial extent of attention, (Owsley et al., 1998). The UFOV has been defined as an attentional window from "which useful information can be acquired without eye and head movements" (Ball et al., 1988; also see Sanders, 1970) and has been found to be predictive of crash risk among older drivers (Sims, McGwin Jr, Allman, Ball, \& Owsley, 2000). Notably, congruent with prominent theories of attention (e.g., Eriksen \& St. James, 1986; LaBerge \& Brown, 1989), the spatial extent of attention as measured by UFOV is 2D, extending only in the horizontal and vertical dimensions (Edwards et al., 2005; Sekuler \& Ball, 1986). However, evidence from experimental studies suggests that the spatial extent of attention is three-dimensional (3D) during driving (Andersen, Ni, Bian, \& Kang, 2011; Pierce, Bian, \& Andersen, 2011). One limitation of those experiments is that the effect of $2 \mathrm{D}$ position was not statistically or experimentally controlled and only statistical controls of 2D size were provided. Therefore, the primary purpose of the current experiment was to test the hypothesis that the effects of $3 \mathrm{D}$ attention are independent of projected 2D size and 2D position on the screen of the simulator. If attention has a spatial extent in depth, then the effects of 3D attention should persist when the projected size 
and position of targets are held constant. Therefore, if the spatial extent of attention is 3D, then tests to assess crash risk that do not assess the scope of attention in depth are potentially limited.

In previous studies, drivers performed a simulated car-following task and detected light changes on banners located above a roadway (Andersen et al., 2011; Pierce et al., 2011). In these studies, depth was simulated by a computer program that provided pictorial and motion information for depth. Drivers were found to have faster reaction times (RTs) in response to light change targets closer in simulated depth than to targets further in simulated depth. However, as a result of linear perspective (a depth cue in which parallel lines converge and size varies as a function of distance), targets simulated as being closer in depth are positioned further from the center of the display in 2D (see Figure 1 for an example scene). Therefore, it is improbable that projected position of the targets accounted for the effects of simulated depth. In addition, as a result of perspective, targets simulated as being closer in depth have larger projected sizes. Andersen et al. (2011) conducted analyses in which they statistically controlled for the effect of size. These results demonstrated that it is improbable that the effects of depth on the RT of drivers are a consequence of changes in 2D projected size resulting from changes in 3D simulated distance. However, those analyses may have had insufficient variability in 2D projected size to provide for a statistically powerful control and did not provide a direct test of the effects of depth independent of projected size. In the present study we examined 3D attention during dual task driving performance when size and location were experimentally manipulated to produce equivalent projected size and location but variations in depth.

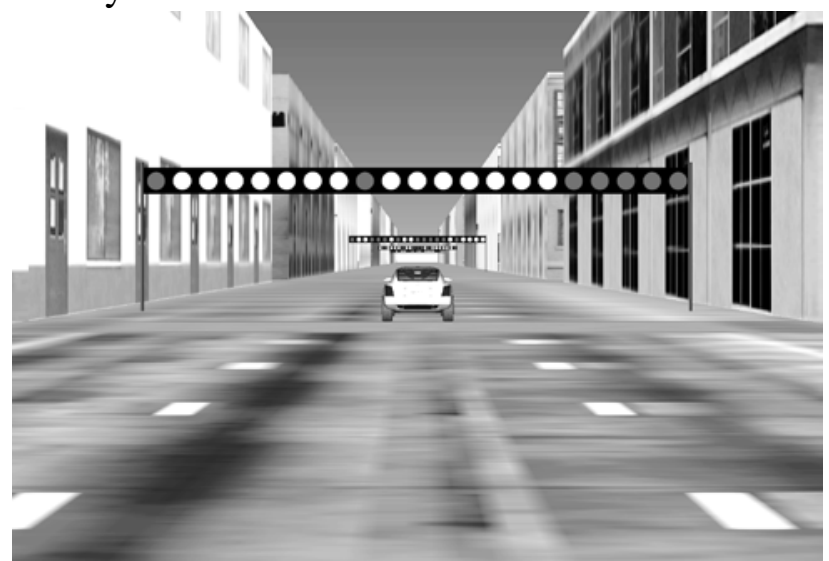

Figure 1. An example driving scene

\section{METHODS}

\section{Participants}

Participants 65 years and older were assigned to the older driver group. All other participants were assigned to the younger driver group. Twenty-four college students, 11 male and 13 female, $(\mathrm{M}=21.75$ years; $\mathrm{SD}=1.03$ years; range 19 - 24 years $)$ and 24 older drivers, 12 male and 12 female ( $\mathrm{M}=73.50$ years; $\mathrm{SD}=4.48$ years; range 66 - 83 years) were paid for their participation. All drivers had normal or corrected-to-normal vision and were naïve to the purpose of the study.

\section{Apparatus}

The driving simulator consisted of a Dell Vostro desktop computer, an ECCI Trackstar 6000 wheel unit and pedal unit, simulation code written in C++ with OpenGL Performer libraries, and 23.1" diagonal LCD monitor with a native resolution of $1280 \times 1024$ pixels and a visual angle of $37.07^{\circ} \times 28.23^{\circ}$ when viewed from $70 \mathrm{~cm}$. The ECCI pedal unit provided closed loop control of the simulator. The controls and the rest of the simulation software updated at $60 \mathrm{~Hz}$. The 
simulation code was modified from a version previously reported by Andersen et al. (2011) and Pierce et al. (2011) to account for updates and modifications to the experimental equipment and procedures. The computer generated 3D scene was of a $15.50 \mathrm{~m}$ wide three-lane one-way road situated with two-story office buildings on both sides. Scene textures were derived from digital photographs of a real vehicle and real buildings, but were digitally altered and rescaled to realistically fit the simulation environment. Asphalt was simulated using a black and white gravel texture pattern. The average luminance of the driving scene was $24.70 \mathrm{~cd} / \mathrm{m}^{2}$. The only vehicle visible in the scene was the lead vehicle, a white sedan initially displayed at a headway distance, i.e. the distance between the drivers' vehicle and the lead vehicle, of $20.50 \mathrm{~m}$. The driver and the lead vehicle were located in the center lane.

Centered above the roadway there was a 13.2m wide array of lights. Each light array had a different random order of 11 red and green lights. Depending on the experimental condition these lights had a small, medium, or large diameter $(41.25 \mathrm{~mm}, 61.88 \mathrm{~mm}$, or $82.50 \mathrm{~mm})$. The simulated heights of the light array were matched to simulated light diameter to allow for the creation of equivalent 2D positions and sizes across 3D conditions $(2.68 \mathrm{~m}, 3.42 \mathrm{~m}$, and $4.16 \mathrm{~m})$. Specifically, large lights viewed with an offset of $4.8 \mathrm{~m}$ at a distance of $60 \mathrm{~m}$ had the same projected size and position as medium lights viewed with an offset of $3.6 \mathrm{~m}$ at a distance of $45 \mathrm{~m}$ and small lights viewed with an offset of $2.4 \mathrm{~m}$ at a distance of $30 \mathrm{~m}$. In addition, large lights viewed with an offset of $2.4 \mathrm{~m}$ at a distance of $60 \mathrm{~m}$ had the same projected size and position as small lights viewed with an offset of $1.2 \mathrm{~m}$ at a distance of $30 \mathrm{~m}$.

In all simulations the speed of the lead vehicle was $60 \mathrm{kph}$ (37.28 mph). During simulator sessions, the speed of the lead vehicle varied according to the sum of three sine waves with equal peak accelerations and decelerations. Specifically, the frequencies of the sine waves were .033, .083 , and $.117 \mathrm{~Hz}$. The phases of the sine waves were restricted such that the speed of the lead vehicle started no more than $+/-1 \mathrm{kph}$ from $60 \mathrm{kph}$. The amplitudes of the sine waves generating lead vehicle speed changes were $15.56 \mathrm{kph}, 6.22 \mathrm{kph}$, and $4.44 \mathrm{kph}$ respectively. This resulted in an average range of speed of $\pm 23.36 \mathrm{kph}(14.51 \mathrm{mph})$ about the mean speed.

\section{Procedure}

The experiment took place in a darkened room. The drivers were seated with their hands on the steering wheel and feet on the pedals as if they were operating a vehicle. When the drivers were ready to start the simulator session, they pulled a paddle behind the steering wheel attached to the steering column. Participants completed four training sessions followed by two experimental sessions. Each session began with the lead vehicle and the drivers' vehicle moving at a constant speed of $60 \mathrm{kph}$ with a constant separation of $20.5 \mathrm{~m}$. Control input (acceleration/deceleration) was not allowed and drivers were instructed to remember this distance as the desired headway distance for the rest of the trial. After 5 seconds drivers heard a tone that indicated the start of the experimental task and the lead vehicle began varying its speed according to the experimental design with drivers asked to maintain following distance.

The first training session familiarized participants with the light detection task. Prior to the session, participants were told that they would not need to use the gas or brake. They were told that their task in this session was to notice when one of the lights in the array turned yellow, 
determine whether it was on the left half of the array or on the right half of the array, and then to pull a response paddle on the corresponding side of the steering wheel. Lights turned yellow when the drivers' vehicle was $30 \mathrm{~m}, 45 \mathrm{~m}$, or $60 \mathrm{~m}$ away from the light array at the $1^{\text {st }}(1.2 \mathrm{~m}), 2^{\text {nd }}$ $(2.4 \mathrm{~m}), 3^{\text {rd }}(3.6 \mathrm{~m})$, or $4^{\text {th }}(4.8 \mathrm{~m})$ light position from the center. Feedback on the light detection task was given by activating a high-tone sound (indicating a correct response); no feedback was given for incorrect responses. If the drivers failed to make a response before passing the light array, then a neutral sound was activated as a reminder. The first training session ended after one minute of task performance. In all sessions with the light detection task, if participants failed to respond, or gave the incorrect response, to four lights, then they repeated the session.

The second training session familiarized participants with the simulated vehicle dynamics and car following task. Prior to the session, participants were told that they did not need to respond to the lights. Participants were told that their task in this session was to use the acceleration and brake pedals to control their speed with the goal of maintaining the same following distance from the lead car that they saw during the first stage of the simulator session. For the second and third training session, the velocity of the lead vehicle was determined by one sine wave function with a frequency of 0.083 and amplitude of $3.889 \mathrm{kph}$. If the headway distance of a participant exceeded 27.3 meters, then a horn sound was activated to simulate an impatient driver behind the drivers' vehicle and to encourage the drivers to attend to the speed variation of the lead vehicle. The second training session ended after one minute of task performance. If participant had a headway distance of $27.3 \mathrm{~m}$ or greater for 15 seconds or if their headway distance was less than $2.35 \mathrm{~m}$, then the participant repeated the session.

The third training session, and all subsequent sessions, required participants to complete the light detection task at the same time as the driving task and terminated after one minute of task performance. In the fourth training session, the speed of the lead vehicle was determined using the sum-of-sines function and terminated after the driver passed all 24 light arrays.

The remaining two sessions were experimental sessions in which the drivers performed both the car following task and the light detection task. Each session contained nine trials. Each trial consisted of 24 light arrays each, with each unique combination of simulated target depth and horizontal position and side (left/right) presented in random order. Each trial lasted for approximately 65 seconds and the duration of the each experimental session, including breaks, was approximately 60 minutes. Participants were instructed to perform both tasks equally well. Participants came back on a second day to complete the second session. The size of the lights was $\mathrm{ABCCBA}$ counterbalanced between trials and the value of $\mathrm{A}, \mathrm{B}$, and $\mathrm{C}$ were counterbalanced between participants.

\section{RESULTS}

Throughout our analyses we reported Greenhouse-Geisser corrected $p$ values, when appropriate, the original degrees of freedom, and the generalized $\eta_{G}^{2}$ effect size. During the experiment, each driver had the opportunity to respond to 432 light changes, 144 light changes per light size. However, the RTs to light changes analyzed in this experient only include the 60 lights for each driver that that had the same 2D projected sizes and positions as other lights, but were at different simulated depths. If participants responded within 300ms of a light change, then their 
performance was near chance $(\mathrm{N}=149$ responses; $\mathrm{M}$ accuracy $=51.01 \%)$. For this reason, responses that occurred in less than 300ms after a light change were excluded from analysis. This criterion excluded no more than $3.33 \%$ of trails for any given participant. To minimize an upward bias in RT as a result of the minimum RT criterion, responses more than $2100 \mathrm{~ms}$ after a light change were also eliminated from analysis. This criterion excluded no more than 3.33\% of trials for any given participant. Trials in which participants failed to respond were removed from analysis. This criterion excluded no more than 3.33\% of trials for any given participant. In the remaning trials, the error rate was below 1\%, so accuracy analyses were not performed. In analyses of RT, trials in which participants gave the incorrect answer were removed from analysis. This criterion excluded no more than $8.33 \%$ of trials for any given participant. In combination, these criteria eliminated no more than $1.91 \%$ of light change data from any analysis and no more than $8.33 \%$ for any given driver.

We conducted a mixed ANOVA to compare RTs of older and younger drivers' for the first group of matched targets, large lights viewed with an offset of $4.8 \mathrm{~m}$ at a distance of $60 \mathrm{~m}(M=805$ $\mathrm{ms} ; S D=281 \mathrm{~ms})$, medium lights viewed with an offset of $3.6 \mathrm{~m}$ at a distance of $45 \mathrm{~m}(M=715$ $\mathrm{ms} ; S D=249 \mathrm{~ms})$, and small lights viewed with an offset of $2.4 \mathrm{~m}$ at a distance of $30 \mathrm{~m}(M=$ $640 \mathrm{~ms} ; S D=180 \mathrm{~ms})$. We found that there were increases in RTs as a function of simulated depth, $F(2,92)=94.03, p<.001, \eta_{G}^{2}=.23$, and faster RTs for younger $(M=641 \mathrm{~ms} ; S D=207$ ms) compared to older drivers $(M=800 \mathrm{~ms}$; $S D=264 \mathrm{~ms}), F(1,46)=45.04, p<.001, \eta_{G}^{2}=.42$, but there was no interaction between these factors, $F(2,92)=.94, p=.39$. Bonferonni adjusted paired samples t-tests comparing RTs between each pair of equivelant positions across depth were all statistically significant, all ps $<.001$.

In addition, we conducted a mixed ANOVA to compare RTs of older and younger drivers' for the second group of matched targets, small lights viewed with an offset of $1.2 \mathrm{~m}$ at a distance of $30 \mathrm{~m}(\mathrm{M}=650 \mathrm{~ms} ; \mathrm{SD}=175 \mathrm{~ms})$ and large lights viewed with an offset of $2.4 \mathrm{~m}$ at a distance of $60 \mathrm{~m}(\mathrm{M}=762 \mathrm{~ms}, \mathrm{SD}=268 \mathrm{~ms})$. We found that there were differences between RTs as a function of simulated depth, $F(1,46)=72.09, p<.001, \eta_{G}^{2}=.18$, and between younger $(M=$ $615 \mathrm{~ms} ; S D=181 \mathrm{~ms})$ and older drivers $(M=800 \mathrm{~ms} ; S D=243 \mathrm{~ms}), F(1,46)=91.05, p<.001$, $\eta_{G}^{2}=.57$, but there was no interaction between these factors, $F(1,46)=1.52, p=.22$.

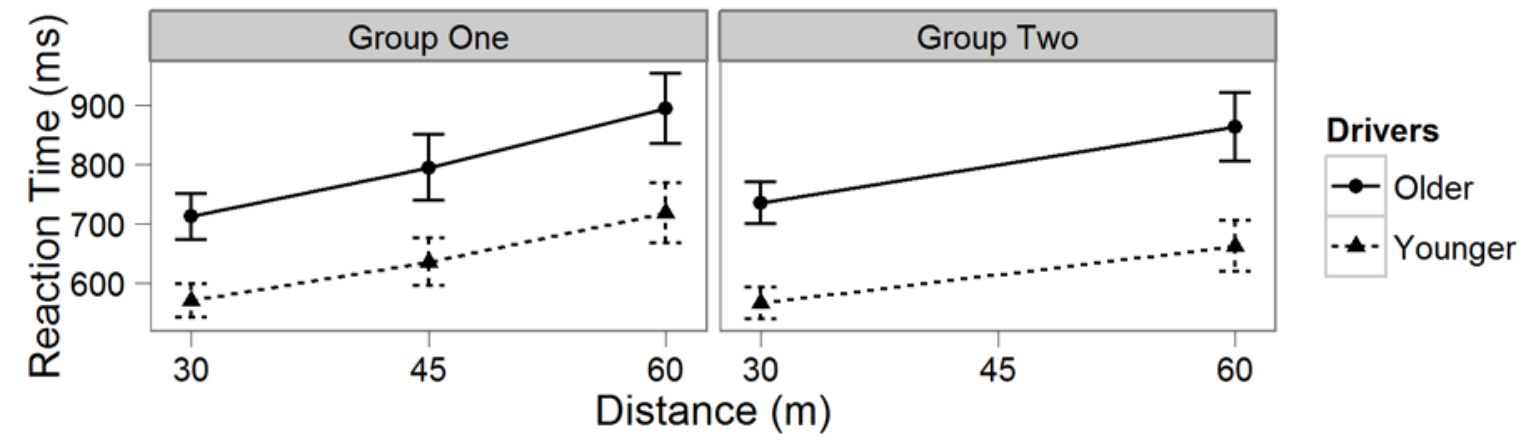

Figure 2. The reaction times of older and younger drivers to groups of light change targets matched in terms of projected 2D positions and sizes as a function of distance. Groups 1 and 2 are for different sets of matched conditions. Error bars represent \pm 1 standard error of the between subjects mean 


\section{CONCLUSION}

The results from this experiment demonstrate although the targets had identical projected 2D positions and sizes, the RTs of drivers to nearer light change targets was faster than their RTs to farther targets. Therefore, we conclude that for both younger and older drivers visual spatial attention is allocated in depth during driving tasks.

In contrast to previous experiments on 3D attention that employed binocular disparity as the primary source of depth information (Andersen, 1990; Atchley, Kramer, Andersen, \& Theeuwes, 1997; Atchley \& Kramer, 2000) and experiments that did not control for projected 2D position and size (Andersen et al., 2011; Downing \& Pinker, 1985; Pierce et al., 2011), the current experiment provided no binocular disparity, size, or projected position information for depth at the moment of the target light change. Instead, targets were identical in projected size and location but varied in simulated depth according to pictorial cues in the scene. The finding of an effect of depth without disparity indicates that 3D attention does not require binocular disparity information (e.g. Cutting \& Vishton, 1995) and that 3D attention effects can be obtained from monocular pictorial depth cues.

Given that depth is an important dimension in driving performance, then an important issue for assessing driving safety is to incorporate the limits of attention in the depth dimensions. Therefore, we recommend that as new procedures are developed to assess spatial attention with the purpose of predicting individual crash risk - that researchers consider including a test of 3D spatial attention to augment already well-established 2D measures such as the UFOV test.

\section{ACKNOWLEDGMENTS}

This work was supported by NIH AG031941 and NIH EY18334

\section{REFERENCES}

Andersen, G. J. (1990). Focused attention in three-dimensional space. Perception \& Psychophysics, 47, 112-120.

Andersen, G. J., Ni, R., Bian, Z., \& Kang, J. (2011). Limits of spatial attention in threedimensional space and dual-task driving performance. Accident Analysis and Prevention, 43(1), 381-90. doi:10.1016/j.aap.2010.09.007

Atchley, P., \& Kramer, A. F. (2000). Age-related changes in the control of attention in depth. Psychology and Aging, 15(1), 78-87.

Atchley, P., Kramer, A. F., Andersen, G. J., \& Theeuwes, J. (1997). Spatial cuing in a stereoscopic display: Evidence for a “depth-aware” attentional focus. Psychonomic Bulletin \& Review, 4(4), 524-529. Retrieved from http://dx.doi.org/10.3758/BF03214343

Ball, K. K., Beard, B. L., Roenker, D. L., Miller, R. K. L., \& Griggs, D. S. (1988). Age and visual search: Expanding the useful field of view. Journal of the Optical Society of America A, 5(12), 2210-2219. doi:10.1364/JOSAA.5.002210 
Cutting, J. E., \& Vishton, P. M. (1995). Perceiving layout and knowing distances: The integration, relative potency, and contextual use of different information about depth. Handbook of perception and Cognition; Perception of space and motion (Vol. 5, pp. 69117). Retrieved from http://wexler.free.fr/library/files/cutting (0) perceiving layout and knowing distances. the integration, relative potency, and contextual use of different information about depth.pdf

Downing, C., \& Pinker, S. (1985). The spatial structure of visual attention. In M. I. Posner \& O. S. M. Marin (Eds.), Attention and Performance XI (pp. 171-187). Hillsdale, New Jersey: Lawrence Erlbaum Associates, Publishers. Retrieved from http://scholar.google.com/scholar?hl=en\&btnG=Search\&q=intitle:The+spatial+structure+of+ visual+attention\#0

Edwards, J. D., Vance, D. E., Wadley, V. G., Cissell, G. M., Roenker, D. L., \& Ball, K. K. (2005). Reliability and validity of useful field of view test scores as administered by personal computer. Journal of Clinical and Experimental Neuropsychology, 27(5), 529-43. doi:10.1080/13803390490515432

Eriksen, C. W., \& St. James, J. (1986). Visual attention within and around the field of focal attention: A zoom lens model. Attention, Perception, \&amp; Psychophysics, 40(4), 225-240. doi:10.3758/BF03211502

LaBerge, D., \& Brown, V. (1989). Theory of attentional operations in shape identification. Psychological Review, 96(1), 101-124.

Owsley, C., Ball, K. K., McGwin, G., Sloane, M. E., Roenker, D. L., White, M. F., \& Overley, E. T. (1998). Visual processing impairment and risk of motor vehicle crash among older adults. Journal of the American Medical Association, 279(14), 1083-1088.

Pierce, R. S., Bian, Z., \& Andersen, G. J. (2011). Age-related limits of 3D spatial attention in dual-task driving. Proceedings of the Sixth International Driving Symposium on Human Factors in Driver Assessment, Training and Vehicle Design (pp. 314-320). Lake Tahoe, CA. Retrieved from http://drivingassessment.uiowa.edu/sites/default/files/DA2011/Papers/046_PierceBian.pdf

Sanders, A. F. (1970). Some aspects of the selective process in the functional visual field. Ergonomics, 13(1), 101-117.

Sekuler, R., \& Ball, K. K. (1986). Visual localization: Age and practice. America, 3(6), 864-867.

Sims, R. V, McGwin Jr, G., Allman, R. M., Ball, K. K., \& Owsley, C. (2000). Exploratory study of incident vehicle crashes among older drivers. Journal of Gerontology, 55(1), M22-M27. doi:10.1093/gerona/55.1.M22

Tefft, B. C. (2008). Risks older drivers pose to themselves and to other road users. Journal of Safety Research, 39(6), 577-82. doi:10.1016/j.jsr.2008.10.002 